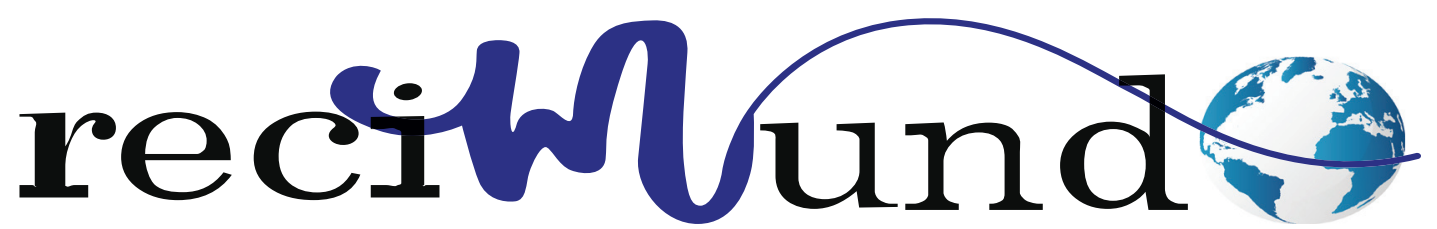

Revista Científica Mundo de la Investigación y el Conocimiento

DOI: 10.26820/recimundo/5.(2).julio.2021.168-187

URL: https://recimundo.com/index.php/es/article/view/1242

EDITORIAL: Saberes del Conocimiento

REVISTA: RECIMUNDO

ISSN: 2588-073X

TIPO DE INVESTIGACIÓN: Artículo de revisión

CóDIGO UNESCO: 32 Ciencias Médicas

PAGINAS: $168-187$

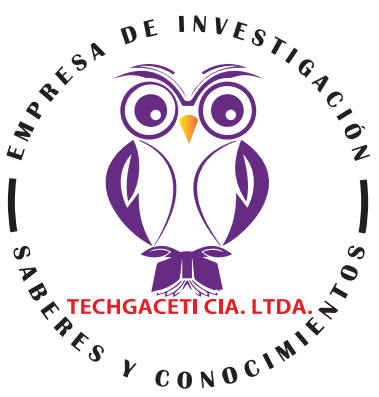

\title{
Uso del material didáctico para la mejora del proceso de enseñanza aprendizaje en medicina
}

Use of didactic material for the improvement of the teaching-learning process in medicine

Uso de material didático para a melhoria do processo de ensino-aprendizagem em medicina

Maritza Alexandra Borja Santillán'; Tibisay Rincón Ríos²; Ofelia Carmen Santos Jiménez;

Ingrid Esmeralda Gurumendi España ${ }^{4}$

RECIBIDO: 11/04/2021 ACEPTADO: 15/06/2021 PUBLICADO: 30/07/2021

1. Doctora en Educación; Magister en Gerencia de Salud para el Desarrollo Local; Especialista en Gerencia y Planificación Estratégica en Salud; Diploma Superior en Desarrollo Local y Salud; Magister en Emergencias Médicas; Medico; Licenciada en Ciencias de la Educación mención Lengua Inglesa y Lingüística; Docente Universidad de Guayaquil, Facultad de Ciencias Médicas, Guayaquil, Ecuador. (DD https://orcid.org/0000-0002-2803-4662

2. Profesora Titular de la Cátedra de Fisiología y Directora de la Escuela de Medicina de LUZ; Universidad del Zulia, ZuliaVenezuela. iD https://orcid.org/0000-0003-4288-1822

3. Doctora en Educación, Docente Principal de la Facultad de Educación Universidad Nacional Mayor de San Marcos, Lima-Perú. Investigadora RENACYT; ofelia.santos@unmsm.edu.pe; (D) https://orcid.org/0000-0003-1294-0641

4. Magister en Gerencia y Administración en Salud; Medico; Universidad de Guayaquil; Guayaquil, Ecuador; ingrid.grumendie@ug.edu.ec; (D) https://orcid.org/0000-0001-6692-719X

CORRESPONDENCIA

Maritza Alexandra Borja Santillán

Guayaquil, Ecuador

๑) RECIMUNDO; Editorial Saberes del Conocimiento, 2021 


\section{RESUMEN}

Sin duda uno de los grandes retos que enfrenta nuestra sociedad actual en el ámbito educacional, es manejar productivamente la dinámica de los procesos de enseñanza aprendizaje, en donde dos grandes generaciones se encuentran en un solo evento. El maestro y el estudiante confluyen en este proceso en busca de un solo objetivo: alcanzar la enseñanza en su máxima expresión. El proceso de enseñanza aprendizaje, a través del uso de material es de vital importancia para el desarrollo cognitivo de las personas y en especial de los estudiantes de medicina, lo cual se ve reflejado no solo en sus vidas académicas sino en el ejercicio de la práctica clínica durante su vida profesional. Se hace un estudio teórico que sustenta la hipótesis de que el proceso de aprendizaje mejora cuando, dentro del proceso educativo se incluyen herramientas didácticas que captan la atención del alumnado mejorando la percepción respecto a la asignatura y el docente. la presente investigación en la recolección de fragmentos de trabajos de investigación, libros y materiales electrónicos sobre los procesos de enseñanza - aprendizaje desde la justificación psicológica hasta las evidencias científicas disponibles al respecto para exponerlas de manera resumida y organizada de forma tal que se puede apreciar el conjunto teórico que cumple con los objetivos trazados.

Palabras clave: Material didáctico, enseñanza, aprendizaje, interacción.

\section{ABSTRACT}

Undoubtedly one of the great challenges facing our current society in the educational field is to productively manage the dynamics of the teaching-learning processes, where two great generations meet in a single event. The teacher and the student come together in this process in search of a single objective: to achieve teaching at its best. The teaching-learning process, through the use of material, is of vital importance for the cognitive development of people and especially medical students, which is reflected not only in their academic lives but also in the exercise of practice. clinic during his professional life. A theoretical study is carried out that supports the hypothesis that the learning process improves when, within the educational process, didactic tools are included that capture the attention of the students, improving the perception of the subject and the teacher. The present investigation in the collection of fragments of research works, books and electronic materials on the teaching - learning processes from the psychological justification to the scientific evidence available in this regard to expose them in a summarized and organized way in such a way that the Theoretical set that meets the objectives set.

Keywords: Didactic material, teaching, learning, interaction.

\section{RESUMO}

Sem dúvida, um dos grandes desafios de nossa sociedade atual no campo educacional é administrar produtivamente a dinâmica dos processos de ensino-aprendizagem, onde duas grandes gerações se encontram em um único evento. O professor e o aluno se reúnem neste processo em busca de um único objetivo: alcançar o ensino no seu melhor. O processo ensino-aprendizagem, através do uso de material, é de vital importância para o desenvolvimento cognitivo das pessoas e especialmente dos estudantes de medicina, o que se reflete não apenas em sua vida acadêmica, mas também no exercício da prática. clínica durante sua vida profissional. É realizado um estudo teórico que suporta a hipótese de que o processo de aprendizagem melhora quando, dentro do processo educativo, são incluídas ferramentas didáticas que captam a atenção dos estudantes, melhorando a percepção do assunto e do professor. A presente investigação na coleta de fragmentos de trabalhos de pesquisa, livros e materiais eletrônicos sobre o ensino - processos de aprendizagem desde a justificação psicológica até as evidências científicas disponíveis a este respeito para expô-los de forma resumida e organizada de tal forma que o conjunto teórico que atende aos objetivos estabelecidos.

Palavras-chave: Material didático, ensino, aprendizagem, interação. 


\section{Introducción}

El aprendizaje es el proceso de asimilación de la información mediante el cual se adquieren nuevos conocimientos, técnicas o habilidades. Las definiciones de aprendizaje se encuentran supeditadas a la perspectiva, tanto del estudiante como del docente. Ausubel (citado en Rodríguez, 2008), define el aprendizaje significativo, como un proceso mediante el cual la nueva información se conecta con algún concepto relevante existente y disponible en la estructura cognitiva del sujeto, lo que hace que el conocimiento adquirido cobre significado; plantea además la existencia del aprendizaje mecáni$\mathrm{co}$, el cual se encuentra relacionado de un modo arbitrario, o cuando el sujeto decide asimilarlo al pie de la letra. Este tipo de aprendizaje imposibilita la integración de los nuevos conocimientos con los ya existentes, es un aprendizaje no relacionado con experiencia, objetos o hechos.

Por su parte, el psicólogo ruso L. Vygotsky, propuso en su teoría constructivista, que el aprendizaje alcanza niveles superiores cuando se da mediatizado por un sujeto cuyo conocimiento o habilidades son superiores a las de sujeto que aprende; en tal virtud el aprendizaje en el sujeto es un proceso social, este no es un proceso individual, sino que inicia desde la relación con lo social y pasa luego a ser un proceso interior que permite el desarrollo del sujeto.

En el nuevo sistema educativo pretende capacitar a los estudiantes para que utilicen con mayor amplitud y seguridad los conocimientos que reciban. (MEC, 2006). La idea principal es valorar más la formación universitaria y la disponibilidad para el empleo de los conocimientos que el dominio de la información. Se trata, en resumidas cuentas, de "enseñar a aprender" para que el estudiante tenga como fin primordial en la Universidad "aprender a aprender". Concibiendo esta etapa educativa como una más del "Aprendizaje a lo largo de toda la vida" debemos conocer que las estrategias son aquellos enfoques y modos de actuar que hacen que el profesor dirija con pericia el aprendizaje de los estudiantes. Las estrategias metodológicas se refieren a los actos favorecedores del aprendizaje Según Duart y Sangrá (2002).

Las estrategias están consideradas como secuencias integradas de procedimientos o actividades elegidas con la finalidad de facilitar la adquisición, almacenamiento y/o utilización de la información. (Díaz Barriga, A. Frida y Hernández R. Gerardo 1999).

El psicólogo Vygotsky expresa que el aprendizaje en el sujeto es un proceso social, este no es un proceso individual sino que inicia desde la relación con lo social y pasa luego a ser un proceso interior que permite el desarrollo del sujeto. Otro aporte lo tenemos en Ausubel quien propuso la teoría del Aprendizaje Significativo, según la cual el sujeto que aprende, relaciona los conceptos a aprender y les da sentido a partir de sus conocimientos previos.

Una estrategia de metodología activa es la forma o manera como los docentes y estudiantes organizan aprendizajes significativos desde la programación de contenidos, la ejecución y la evaluación hasta la organización de los ambientes de aprendizaje, estructuración y utilización de materiales educativos y uso óptimo de los espacios y tiempos del aprendizaje.

La evolución educativa nos exige adaptarnos a las necesidades de nuestro entorno educativo, impulsándonos a ejecutar procesos no convencionales a fin de llegar a los discentes. Manejar adecuadamente los materiales didácticos, ya sean convencionales o a través de técnicas multimedias e interactivas, mejorará la comprensión de la información o conocimiento por parte de los estudiantes, precisando sus objetivos en su trabajo individual y potenciando el desarrollo de actitudes como responsabilidad y trabajo en equipo. 
Se pretende realizar una justificación teórica que permite impulsar el uso de material didáctico en el proceso de enseñanza, sobretodo en la educación superior en donde se puede identificar que se pierde la practica de estas herramientas.

\section{Metodología}

La metodología se puede definir como un conjunto organizado de técnicas y procedimientos tendientes a desarrollar la transmisión de conocimientos, de la manera más fácil en función de los objetivos y competencias preestablecidas.

Es por ellos que hemos basado la presente investigación en la recolección de fragmentos de trabajos de investigación, libros y materiales electrónicos sobre los procesos de enseñanza - aprendizaje desde la justificación psicológica hasta las evidencias científicas disponibles al respecto para exponerlas de manera resumida y organizada de forma tal que se puede apreciar el conjunto teórico que cumple con los objetivos trazados.

\section{Resultados}

\section{Proceso de enseñanza-aprendizaje}

Chadwick (1979) apunta que la expresión de capacidades y de características psicológicas del estudiante, desarrolladas y actualizadas a través del proceso de enseñanza-aprendizaje, le posibilita obtener un nivel de funcionamiento y logros académicos a lo largo de un período, año o semestre, que se sintetiza en un calificativo final (cuantitativo en la mayoría de los casos) evaluador del nivel alcanzado.

El aprendizaje ha sido estudiado desde múltiples perspectivas y por diferentes autores. Siguiendo la línea de estudios e investigaciones, encontramos la teoría sociocultural de Vigotsky que indica que el desarrollo del ser humano está íntimamente ligado con su interacción en el contexto socio histórico. Esto conlleva al análisis de las implicaciones educativas de dicha teoría en los procesos de enseñanza y aprendizaje. De acuerdo con Vigotsky (1977) el aprendizaje precede al desarrollo. El autor distingue dos niveles en el desarrollo: el desarrollo real que indica lo alcanzado por el individuo y el desarrollo potencial que muestran lo que le individuo puede hacer con la ayuda de los demás a lo que se ha denominado zona de desarrollo próximo.

Éste concepto es básico para los procesos de enseñanza y aprendizaje pues la educadora y el educador debe tener en cuenta el desarrollo del estudiante en sus dos niveles para promover en los niveles de avance y autorregulación mediante actividades, como lo proponía Vigotsky. (Carreño, s.f, pág. 35). Por otro lado, se define el aprendizaje por descubrimiento, donde todo conocimiento real es aprendido por uno mismo. En base a esto propone la teoría de la instrucción que "se ocupa de la forma en que lo que uno desea enseñar pueda ser mejor aprendido, de mejorar más que describir el aprendizaje" (Brunner, 1969). Tenemos a Bandura (1977) y otros teóricos que postulan el aprendizaje social, un proceso cognitivo basado en la observación de un modelo o de instrucciones.

Se ha descrito como se ha estudiado el aprendizaje desde diversos paradigmas, autores y cada uno de sus aportaciones al proceso, lo que ha llevado a demostrar que el estudio del aprendizaje siempre tomará diferentes direcciones. Las definiciones de aprendizaje se encuentran supeditadas a la perspectiva desde la que se estudian en la presentación y explicación de diversas perspectivas un referente tradicional Mayer (1992). Este autor realizó una comparación entre las perspectivas de aprendizaje $Y$ distinguió dos: la conductual y la cognitiva. Mayer (1992) mantiene que hay un cambio de paradigma en la psicología de la educación obteniendo las tres denominadas

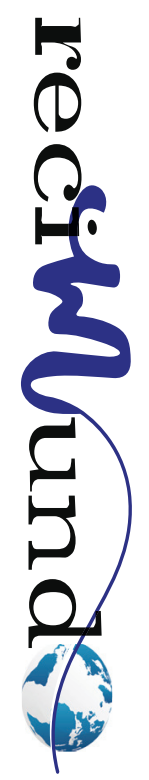


metáforas de aprendizaje: (1) aprendizaje como adquisición de respuestas, (2) aprendizaje como adquisición de conocimiento y (3) aprendizaje como construcción del significado, las dos últimas metáforas están incluidas en la perspectiva cognitiva.

1. Aprendizaje como adquisición del conocimiento, aquí el estudiante es un procesador de información, que solo se limita a receptar y el profesor se convierte en el sujeto que ofrece la información.

2. Aprendizaje como construcción de conocimiento, esta segunda concepción cognitiva del aprendizaje surgió alrededor de los ochenta y definió en el aprendizaje como la construcción por parte del aprendiz de su propio conocimiento con la ayuda del profesor. La enseñanza por tanto se centra en los procesos cognitivos del aprendiz.

Numerosos teóricos han desarrollado teorías sobre el aprendizaje humano y se puede agrupar en tres movimientos uno de ellos me procedencia principalmente europea se refiere al movimiento constructivista que integra la Teoría Genética (Piaget, 1929); La Teoría Sociocultural (Vygotsky, 1962) y Aprendizaje Significativo (Ausubel, 1963).

Susacasa (2013), expresa que la programación de la enseñanza y la elaboración de los materiales pedagógicos, deben ser realizadas por el docente como una forma de administrar los recursos orientados a lograr el aprendizaje en los estudiantes, para la formación profesional en medicina. De igual manera es indispensable tomar en cuenta que el material didáctico elaborado, debe estar científicamente diseñado, promoviendo el aprendizaje autónomo y responsable hacia la labor médica.

La integración de los recursos didácticos en la educación debe ser diseñada, específicamente, para el aprendizaje, el mismo que potencie el conocimiento de manera directa.
Rivas (2014), manifiesta en cuanto al aprendizaje en medicina, por ejemplo, que la simulación y el modelado han sido técnicas que permiten al estudiante acceder a diferentes partes del cuerpo y sus funciones en un ambiente controlado sin faltar a la ética, puesto que no existe el contacto directo ni manipulación del paciente ni de animales.

Arroyo (2014) expresa que los simuladores constituyen una herramienta pedagógica que permiten acortar tiempo y distancia, facilitando el acceso a la problemática a estudiar de forma virtual, permitiendo al estudiante proponer alternativas y soluciones a partir de un proceso de aprendizaje autorregulado; este autor cita a Pérez (2011), de quien recoge los aspectos fundamentales de la simulación, las cuales son:
"a) la posibilidad de tiempo real, que permite enfocar la línea de actuación y moverse dentro de un escenario virtual, b) la inmersión producida por la profundidad del mundo vir- tual y c) el nivel de interacción que integra con todos los ele- mentos de una situación"

Piña (2010), refiere que el uso de material didáctico facilita al docente el acercamiento con sus estudiantes para el logro del aprendizaje en ellos. El material didáctico consiste en todo aquel objeto artificial y/o natural que posibilita al estudiante acercarse a la realidad y al contenido de aprendizaje, dado que la actividad docente consiste en la mediación entre la cultura y el alumno. Hay que tomar en cuenta que el material que se utiliza en aprendizaje debe estar elaborado o utilizado con fines netamente didácticos para poder lograr los objetivos planteados, entre ellos se cuentan los escenarios de aprendizaje, los medios audiovisuales y el material bibliográfico.

Los materiales didácticos para procesos educativos, está relacionado con el desa- 
rrollo de materiales para facilitar el aprendizaje de los estudiantes, siempre y cuando, los recursos didácticos utilizados, cumplan con su objetivo planteado dentro de la planificación académica.

Los recursos didácticos corresponden a elementos que el docente adapta o modifica según el objetivo de aprendizaje que desea lograr de sus estudiantes, pudiendo hacer más fácil su tarea de mediación en el aprendizaje a la vez que facilita la comprensión y asimilación de los contenidos al alumno permitiendo despertar y captar su interés, a la vez que potencia sus capacidades creadoras, dado que acercan al estudiante a la realidad. Estos materiales pueden ser convencionales, audiovisuales y tecnológicos. (Sánchez, 2010).

El investigador apunta a una innovación en las estrategias de enseñanza aprendizaje, con la finalidad de que los educandos se interesen en lo se está enseñando, enfocando el aprendizaje a la realidad.

\section{Uso del Material Didáctico}

Estrategias de Aprendizaje son las que corresponden a acciones voluntarias, conscientes, secuenciadas y flexibles que son utilizadas por el sujeto con el propósito de facilitar y consolidar el aprendizaje o la resolución de problemas. Estos procedimientos permiten la adquisición, almacenamiento y recuperación de la información dando paso a la acomodación y asimilación del nuevo conocimiento con el conocimiento previo (Garzuzi \& Mafauad, 2014) (León, Risco, \& Alarcón, 2014).

El uso de las diferentes estrategias de aprendizaje ocurre en asociación con otros procesos y recursos cognitivos que posee el sujeto o estudiante. Estos otros recursos son:

- Procesos cognitivos básicos: son las operaciones y procesos para el pro- cesamiento de la información como la atención, la percepción, codificación, almacenaje, mnémicos, recuperación, etc., están presentes desde la infancia.

- Conocimientos conceptuales específicos: son los conocimientos específicos de determinados temas que poseemos con anticipación y que se encuentran organizadas de forma jerárquica en nuestro bagaje cognitivo.

- Conocimiento estratégico: esto corresponde específicamente a las estrategias de aprendizaje y es lo que se conoce como saber cómo conocer (Díaz-Barriga \& Hernández, 2010).

- Conocimiento meta-cognitivo: es el conocimiento que tenemos sobre qué y cómo lo sabemos, es decir el conocimiento que poseemos sobre nuestros propios procesos, operaciones y características cognitivas.

Estos tipos de conocimiento acompañan siempre de forma enlazada al uso de las estrategias de aprendizaje.

Las estrategias de aprendizaje están clasificadas por lo generales o específicas que son, en función del dominio del conocimiento al que aplican, por el tipo de aprendizaje que persiguen (asociación o reestructuración), por su finalidad, las técnicas que abarcan, entre otras.

Siguiendo la clasificación de Román Sánchez y Gallego Rico (2001; Garzuzi \& Mafauad, 2014) tenemos lo siguiente:

- Estrategias de adquisición de la información: la atención es el primer elemento necesario para adquirir la información, de tal manera que es la vía de selección, transformación y transmisión de la información desde el ambiente hasta el registro sensorial.

- Dentro de los procesos atencionales encontramos las estrategias de exploración que consiste en leer repetidamente el material verbal, y la fragmentación que

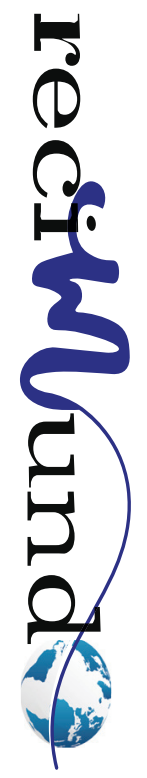


consiste en técnicas como el subrayado lineal e idiosincrático, y el epigrafiado.

- En segundo lugar, encontramos los procesos de repetición, que, junto a la atención, llevan la información hasta la memoria a corto plazo. Dentro de estos procesos encontramos técnicas como el repaso reiterado en voz alta o metal.

- Estrategias de codificación de la información: estas permiten pasar la información de la memoria a corto plazo a la memoria a largo plazo en un proceso en el que se integran los conocimientos previos con los actuales formando nuevos esquemas cognitivos.

- Entre las estrategias de codificación tenemos en primer lugar las nemotecnias, que constituyen una forma de organización superficial de la información, aquí tenemos las rimas, palabras clave, etc. En segundo lugar, encontramos las estrategias de elaboración que permiten hacer asociación de la información, entre ellas tenemos las comparaciones, metáforas, imágenes, etc.

- Finalmente tenemos las estrategias de organización, por medio de las cuales el conocimiento llega a ser significativo y manejable, aquí se cuentan técnicas como los agrupamientos, secuencias, mapas y diagramas.

- Estrategias de recuperación de la información: estas estrategias permiten recuperar la información desde la memoria a largo plazo, entre ellas están las de búsqueda que dependen de la organización de la información en la memoria, aquí están las codificaciones como las metáforas y los mapas, y los indicios entre los que encontramos las palabras clave, conjuntos, etc.; otra estrategia de recuperación de la información es la de generación de respuestas que permiten la adaptación positiva de una conducta, como la libre asociación y la ordenación.

- Estrategias de apoyo a la información: estas estrategias son un complemento de las estrategias anteriores y se subdividen en meta-cognitivas, afectivas y sociales.

- Las estrategias meta-cognitivas permiten al estudiante que cumpla con sus metas de aprendizaje cumpliendo los objetivos trazados y modificando estrategias si fuera necesario; aquí entran el autoconocimiento, la planificación, la regulación y la evaluación.

- Las estrategias afectivas están relacionadas con el estado anímico que interfiere en el proceso de aprendizaje; entre ellas contamos el autocontrol y las autoinstrucciones con las cuales se actúa directamente sobre la autoestima, la ansiedad, la autoeficacia, entre otras.

Las estrategias sociales posibilitan al estudiante controlar situaciones ambientales como resolución de conflictos y el trabajo colaborativo. $Y$ finalmente las estrategias motivacionales son las que caracterizan la inclinación regular o momentánea hacia el estudio, entre ellas están las motivaciones intrínsecas o extrínsecas.

\section{Estrategias de enseñanza}

La actividad docente implica todos aquellos procedimientos estratégicos que tienen como finalidad que el estudiante pueda asignar significado a determinado conocimiento, mejorando sus habilidades cognitivas; se puede definir las estrategias de aprendizaje como los procedimientos y recursos que el docente emplea para generar en el estudiante un aprendizaje significativo, lo cual implica una continua revisión de dichos recursos y procedimientos para realizar las modificaciones a la estructura y/o contenido del material utilizado que promueva en el aprendiz la capacidad de escoger y manejar sus propios procedimientos para internalizar el conocimiento de manera autónoma y significativa (Díaz-Barriga \& Hernández, 2010).

Para que el docente pueda poner en marcha cualquier tipo de estrategia metodológica para el manejo de su clase, es necesario 
que previamente conozca las características particulares cognitivas y de aprendizaje de cada uno de sus estudiantes; esto le permitirá modificar sus propios estilos de enseñanza y poder utilizar las estrategias más adecuadas para lograr involucrar a sus discentes en el proceso de aprendizaje (Yancen, Consuegra, Herrera, Pacheco, \& Díaz, 2013) (Gravini, Cabrera, Ávila, \& Vargas, 2009).

Tabla 1. Estilos de aprendizaje VS Estrategias educativas

\begin{tabular}{|c|c|}
\hline $\begin{array}{ll}\text { Estilos } & \text { de } \\
\text { aprendizaje } & \end{array}$ & ESTRATEGIAS EDUCATIVAS \\
\hline REFLEXIVO & $\begin{array}{l}\text { Grupo pequeño, mímicas, escenificaciones, escritos, } \\
\text { creativos (poesía, cantos, historias, parábolas), } \\
\text { afiches, collages, esculturas, dibujos, boletines, } \\
\text { decoración, proyectos artísticos, narrativa, etc. }\end{array}$ \\
\hline ACTIVO & $\begin{array}{l}\text { Dramas, estudio de casos, actividades manuales, } \\
\text { simulaciones, prueba de situaciones y teorías, } \\
\text { situaciones de enfermería, organizar programas, etc. }\end{array}$ \\
\hline PRAGMÁTICO & $\begin{array}{l}\text { Debate, deducciones, planificación de dramas, } \\
\text { planificación de experimentos, resolución de } \\
\text { problemas, aplicación de lecciones al diario vivir, } \\
\text { inventarios personales, mesas redondas, reseña, etc. }\end{array}$ \\
\hline TEÓRICO & $\begin{array}{l}\text { Clase tradicional, seminarios, clases magistrales, } \\
\text { lecturas organizadas, demostraciones, investigación, } \\
\text { preguntas, cuestionarios, crucigramas, etc. }\end{array}$ \\
\hline
\end{tabular}

Fuente: (Yancen, Consuegra, Herrera, Pacheco, \& Díaz, 2013, pág. 408)

Según lo que se puede apreciar en la Figura 4, las estrategias que cada docente utiliza están también directamente relacionadas con la propia forma de aprender que tiene el mismo docente, lo que caracteriza su estilo de enseñanza. Sin embargo, el éxito de su clase dependerá de su ajuste a los estilos de aprendizaje de sus estudiantes (Yancen, Consuegra, Herrera, Pacheco, \& Díaz, 2013).

La enseñanza es un proceso de apoyo para que el estudiante construya su propio conocimiento, todo lo que el docente realiza dentro de su relación con el estudiante, constituye un andamiaje del proceso de aprendizaje. Esta relación bilineal entre do- cente y estudiante hace que el aprendizaje sea enriquecedor en ambas vías, puesto que la realidad de cada clase no resulta ser siempre como lo planificado, esto hace que la aportación entre ambas partes sea mucho más real, consciente y espontánea en cada ocasión (Díaz-Barriga \& Hernández, 2010).

El amplio repertorio de estrategias de enseñanza que los docentes utilizan y aplican para generar entre sus estudiantes aprendizajes significativos, tiene diversas clasificaciones, entre las que se cuentan:

- Objetivos o propósitos del aprendizaje

- Resúmenes

- Ilustraciones

- Organizadores previos

- Preguntas intercaladas

- Pistas topográficas y discursivas

- Analogías

- Mapas conceptuales y redes semánticas

- Uso de estructuras textuales

Es necesario identificar que las estrategias pueden utilizarse ya sea antes (preinstruccionales), durante (coinstruccionales) o después (posinstruccionales) de un contenido de aprendizaje, por lo que tendríamos la siguiente clasificación: 
Tabla 2. Estrategias de enseñanza.

\begin{tabular}{|c|c|}
\hline \multicolumn{2}{|r|}{ Estrategias de enseñanza } \\
\hline Objetivos & $\begin{array}{l}\text { Enunciado que establece condiciones, tipo de actividad } \\
\text { y forma de evaluación del aprendizaje del alumno, } \\
\text { generación de expectativas apropiadas en los alumnos. }\end{array}$ \\
\hline Resumen & $\begin{array}{l}\text { Sintesis y abstracción de la información relevante de un } \\
\text { discurso oral o escrito. Enfatiza conceptos clave, } \\
\text { principios, término y argumento central. }\end{array}$ \\
\hline Organizador Previo & $\begin{array}{l}\text { Información de tupo introductorio y contextual. Es } \\
\text { elaborado con un nivel superior de abstracción, } \\
\text { generalidad e inclusividad, que la información que se } \\
\text { aprenderá tiende un puente cognitivo entre la } \\
\text { información nueva y la previa. }\end{array}$ \\
\hline Ilustraciones & $\begin{array}{l}\text { Representación visual de los conceptos, objetos o } \\
\text { situaciones de una teoria o tema especifico (fotografia, } \\
\text { dibujos, esquemas, graficas, dramatizaciones, etcétera). }\end{array}$ \\
\hline Analogías & $\begin{array}{l}\text { Proposición que indica que una cosa o evento (concreto } \\
\text { y familiar) es semejante a otro (desconocido y abstracto } \\
\text { o complejo). }\end{array}$ \\
\hline $\begin{array}{l}\text { Preguntas } \\
\text { Intercaladas }\end{array}$ & $\begin{array}{l}\text { Preguntas insertadas en la situación de enseñanza o en } \\
\text { un texto. Mantienen la atención y favorecen la práctica, } \\
\text { la retención y la obtención de información relevante. }\end{array}$ \\
\hline $\begin{array}{l}\text { Pistas Topográficas y } \\
\text { Discursivas }\end{array}$ & $\begin{array}{l}\text { Señalamiento que se hace en un texto o en la situación } \\
\text { de enseñanza para enfatizar y/u organizar elementos } \\
\text { relevantes del contenido por aprender. }\end{array}$ \\
\hline $\begin{array}{l}\text { Mapas Conceptuales } \\
\text { y Redes Semánticas }\end{array}$ & $\begin{array}{l}\text { Representación gráfica de esquemas de conocimientos } \\
\text { (indican conceptos, proposiciones y explicaciones) }\end{array}$ \\
\hline $\begin{array}{l}\text { Uso de estructuras } \\
\text { textuales }\end{array}$ & $\begin{array}{l}\text { Organizaciones retóricas de un discurso oral o escrito, } \\
\text { que influyen en su comprensión y recuerdo. }\end{array}$ \\
\hline
\end{tabular}

Fuente: (Díaz-Barriga \& Hernández, 2010, pág. 142)

Las estrategias preinstruccionales son las que preparan al estudiante acerca del cómo y qué va a aprender, estableciendo conexión entre los conocimientos previos para fundamentar el aprendizaje actual; aquí están los objetivos y los organizadores previos.

Las estrategias coinstruccionales son aquellas que apoyan el contenido de aprendizaje identificando ideas principales, contenidos y estructura de los contenidos; aquí encontramos ilustraciones, redes semánticas, mapas conceptuales, analogías, etc.

Finalmente, las estrategias posinstruccionales representan aquellas que se utilizan al final del contenido de aprendizaje y sirven como herramientas para sintetizar la información recibida y formar una representación integral y crítica de la misma; entre ellas están los resúmenes, cuestionarios, mapas conceptuales, etc.

Las estrategias de aprendizaje están también clasificadas de acuerdo a los procesos cognitivos que promueven en el aprendizaje de los estudiantes, según se puede ver en la siguiente figura.

Tabla 3. Estrategias de aprendizaje según el proceso cognitivo

\begin{tabular}{|l|ll|}
\hline $\begin{array}{l}\text { Proceso cognitivo en el que } \\
\text { incide la estrategia }\end{array}$ & Tipos de estrategia de enseñanza \\
\hline $\begin{array}{l}\text { Activación de conocimientos } \\
\text { previos }\end{array}$ & $\begin{array}{l}\text { Objetivos o propósitos Pre- } \\
\text { interrogantes }\end{array}$ \\
\hline $\begin{array}{l}\text { Generación de expectativas } \\
\text { apropiadas }\end{array}$ & $\begin{array}{l}\text { Actividad de generadora de } \\
\text { información previa }\end{array}$ \\
\hline $\begin{array}{l}\text { Orientar y mantener la atención } \\
\text { Ilustraciones } \\
\text { Pistas o claves tipográficas o } \\
\text { discursivas }\end{array}$ \\
\hline $\begin{array}{l}\text { Promover una organización más } \\
\text { ha de aprender (mejorar las } \\
\text { conexiones internas) }\end{array}$ & $\begin{array}{l}\text { Mapas conceptuales } \\
\text { Redes Semánticas }\end{array}$ \\
\hline $\begin{array}{l}\text { Para potenciar el enlace entre el } \\
\text { conocimiento previo u la información que se } \\
\text { información que se ha de aprender } \\
\text { (mejorar las conexiones externas) }\end{array}$ & Organizadores previos analógicos \\
\hline
\end{tabular}

Fuente: (Díaz-Barriga \& Hernández, 2010, pág. 145)

Cada estrategia tiene como finalidad ayudar a que el estudiante alcance un aprendizaje significativo, por lo tanto, estas estrategias pretender logran un efecto durante el proceso cognitivo de aprendizaje, tal como se muestra. 
Tabla 4. Estrategias y efectos esperados en el aprendizaje de los estudiantes

\begin{tabular}{|c|c|}
\hline $\begin{array}{ll}\text { Estrategias } & \text { de } \\
\text { Enseñanza } & \end{array}$ & Efectos esperados en el alumno \\
\hline Objetivos & $\begin{array}{l}\text { Conoce la finalidad y alcance del material y } \\
\text { cómo manejarlo } \\
\text { El alumno sabe que se espera de él al terminar de } \\
\text { revisar el material } \\
\text { Ayuda a la contextualizar sus aprendizajes y a } \\
\text { darles sentido. }\end{array}$ \\
\hline Ilustraciones & Facilita la codificación visual de la información \\
\hline $\begin{array}{l}\text { Preguntas } \\
\text { intercaladas }\end{array}$ & $\begin{array}{l}\text { Permite practicar y consolidar to que se ha } \\
\text { aprendido } \\
\text { Resuelve sus dudas } \\
\text { Se autoevalúa gradualmente }\end{array}$ \\
\hline Pistas tipográficas & $\begin{array}{l}\text { Mantiene su atención e interés } \\
\text { Detecta información principal } \\
\text { Realiza codificación selectiva }\end{array}$ \\
\hline Resúmenes & $\begin{array}{l}\text { Facilita el recuerdo y la comprensión de la } \\
\text { información relevante del contenido que se ha de } \\
\text { aprender }\end{array}$ \\
\hline $\begin{array}{l}\text { Organizadores } \\
\text { previos }\end{array}$ & $\begin{array}{l}\text { Hace más accesible y familiar el contenido } \\
\text { Elabora una visión global y contextual }\end{array}$ \\
\hline Analogías & $\begin{array}{l}\text { Comprende información abstracta } \\
\text { Traslada lo aprendido a sus ámbitos }\end{array}$ \\
\hline $\begin{array}{l}\text { Mapas conceptuales y } \\
\text { redes semánticas }\end{array}$ & $\begin{array}{l}\text { Realiza una codificación visual y semántica de } \\
\text { conceptos, proposiciones y explicaciones } \\
\text { Contextualiza las relaciones entre conceptos y } \\
\text { proposiciones }\end{array}$ \\
\hline Estructuras textuales & $\begin{array}{l}\text { Facilita el recuerdo y la comprensión de lo más } \\
\text { importante de un texto }\end{array}$ \\
\hline
\end{tabular}

Fuente: (Díaz-Barriga \& Hernández, 2010, pág. 148)

\section{Materiales didácticos}

Los recursos didácticos son los medios materiales de apoyo que van a ser utilizados por el docente o maestro para mediar los contenidos de aprendizajes significativos nuevos o de refuerzo mediante la construcción del conocimiento por los propios estudiantes. El docente debe utilizar recursos didácticos activos y funcionales, innovadores, creativos y constructivos desde los propios estudiantes mediante aprendizaje, de interacción entre compañeros y compañeras. Según las distintas actividades o materias, podemos considerar los siguientes materiales: Materiales de juego, Materiales de lenguaje, Materiales de educación sen- sorial, Materiales de matemáticas, Materiales de observación y experimentación.

Existen materiales didácticos de piezas anatómicas tratadas con Glicerinización y tratadas con formol; en el caso de las piezas con formol se presentan los siguientes efectos secundarios como en el caso de la irritación de la nariz y garganta, dificultad respiratoria, bronquitis sensible y su clasificación como carcinógeno humano. Esto permitió plantear una nueva forma para poder conservar y así llevar a cabo la elaboración varios materiales que van a servir de herramienta didáctica para poder facilitar los procesos de enseñanza y aprendizaje en los estudiantes y docentes. En tal virtud, en el presente estudio, se ha utilizado materiales didácticos de piezas anatómicas tratadas con glicerina, para lo cual se detalla a continuación el proceso de Glicerinización:

\section{Proceso de enseñanza aprendizaje}

Las teorías del aprendizaje han sido concebidas desde la psicología con diferentes enfoques que abarcan las características de aprendizaje del estudiante, el entorno de aprendizaje y el docente. Estas teorías recaen sobre dos grandes familias que abarcan en su seno, propuestas diversas del proceso de aprendizaje: conductismo y cognitivismo.

\section{Conductismo}

Las teorías conductistas parten del esquema estímulo-respuesta como postulado base del proceso de aprendizaje, haciendo referencia a la asociación de respuestas desencadenadas por un estímulo; tienen aquí especial importancia la experiencia, la repetición de respuestas y los reforzadores, sean estos positivos o negativos (Facundo, 2013). Esta escuela se fundamenta en la filosofía asociacionista iniciando por las ideas de Aristóteles quien especificara los conceptos en lo que se refiere a cómo los sujetos recuerdan; estos son los conceptos

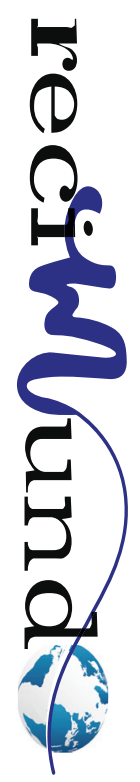


de continuidad (todo lo que existe tiene un antecedente que se ha formado por medio de la experiencia sensible) y analogía (el mismo objetivo puede alcanzarse por estímulos o vías diferentes). De esta forma quedan establecidos el empirismo y asociacionismo como una vía de explicación del funcionamiento del psiquismo.

David Hume expresa que la causalidad surge por las ideas simples que se asocian entre sí para formar las ideas complejas a través de la actividad de la mente; esta relación entre experiencia y asociación de ideas, forman la causalidad. Otros asociacionistas como Locke y Berkeley afirman que la realidad existe por medio de la vía sensorial. Kant por su parte establece que el conocimiento científico es posible a través de lo que nuestro entendimiento pone en los objetos; es decir, el conocimiento a priori de los objetos, lo cual establece la propia acción de conocer como resultado del objeto real y de la reflexión del mismo. Así, la causalidad es una forma a priori de conocimiento en la que se suceden los fenómenos de la experiencia resultando en la representación del objeto conocido (Romero, 2012).

A partir de estas afirmaciones, la relación causa y efecto pasó a ser premisa de las ciencias; así la escuela conductista propone también que la explicación científica del comportamiento humano parte de una relación innegable de causa y efecto: estímulo-respuesta, que surge a partir de los estudios realizados por I. Pavlov del reflejo condicionado (Facundo, 2013).

Las teorías conductistas que influyeron en la educación fueron:

Teorías conexionistas: representada por Edward Thorndike, refiere que el aprendizaje se da por asociación entre un estímulo sensorial y la respuesta que este desencadena; el ensayo y error es la base del aprendizaje, lo cual implica una selección y conexión de respuestas acertadas. Thorndike plantea leyes y principios que explican el mecanismo de aprendizaje, las cuales son:

a) Ley del ejercicio: corresponde a la formación de hábitos a través de la conexión de estímulo-respuesta mediante la práctica o el debilitamiento de la misma.

b) Ley del efecto: corresponde al fortalecimiento de las consecuencias de las respuestas emitidas; así, si una acción es seguida de una recompensa entonces la conducta se refuerza, pero si, por el contrario, a la conducta emitida le sigue un factor perturbador, entonces la conexión estímulo-respuesta se debilita.

Dentro de la práctica educativa la teoría de Thorndike se lleva a cabo por ejemplo en el uso de estímulos visuales, premios, o la repetición como vía de aprendizaje.

Condicionamiento clásico: John Watson es quien crea esta teoría partiendo de los estudios de Pavlov quien propuso el concepto de reflejo condicionado; de esta forma Watson expresa que nuestras acciones son el resultado de reflejos condicionados, por asociación de estímulos condicionados. Se proponen aquí dos principios para el aprendizaje:

- Principio de frecuencia: mientras más se repite una respuesta frente a un estímulo determinado, mayor es la posibilidad de que la conducta se repita frente al mismo estímulo.

- Principio de reiterancia: cuanto más reciente sea la respuesta frente a un estímulo, mayor probabilidad hay de que la misma respuesta se utilice frente al mismo estímulo

1. Condicionamiento con refuerzo: las teorías de Hull y Skinner son representativas en cuanto a los reforzadores de la conducta. Hull expresa que entre el estímulo y la conducta se encuentran las variables independientes que son las que de alguna ma- 
nera condicionan la respuesta (conducta); estas variables intervinientes son los hábitos, el impulso y la motivación. Por su parte B. Skinner manifiesta que el aprendizaje es condicionamiento, por tanto, si queremos que una persona emita determinada conducta, es necesario reforzar positivamente dicha conducta con estímulos que resulten gratificantes; así mismo, si deseamos que una conducta se extinga, entonces es necesario disminuir el reforzador o reforzar negativamente.

\section{Cognitivismo}

La psicología cognitiva hace referencia a los procesos en los que una información sensorial que ingresa es analizada, transformada, elaborada, almacenada, recuperada y utilizada, de forma que los códigos cognitivos posibilitan los procesos mentales. Así, los procesos mentales o cognitivos permiten crear códigos cognitivos que son útiles a los sujetos diariamente en situaciones como trabajar, analizar, tomar decisiones, jugar, solucionar problemas, etc. (Neufeld, Georgen, \& Milnitsky, 2011), por medio de la memoria, percepción, atención, conciencia, reconocimiento de objetos, desarrollo cognitivo, lenguaje y representación del conocimiento (Fierro, 2011).

El cognitivismo sienta sus bases en la teoría filosófica del racionalismo, dado que el sujeto conoce por medio de elaboraciones cognitivas del mundo que lo rodea, de modo que el comportamiento del sujeto no es resultado de un estímulo sino de una representación de la realidad que se percibe (Hernández \& Alonso, 2012). De esta forma el ser humano no solamente recibe información y emite una respuesta, sino que además procesa dicha información y emite información nueva, producto de las estructuras y esquemas mentales por medio de los cuales interpreta su mundo.

A pesar de la influencia innatista del racionalismo cartesiano, el cognitivismo abarca la teoría constructivista según la cual los sujetos construyen su conocimiento a partir de la reorganización continua de la información recibida del entorno, de esta forma, la continua relación con el mundo externo permite al sujeto reelaborar y resignificar continuamente la realidad (Hernández \& Alonso, 2012).

Entre las teorías cognoscitivas más representativas podemos mencionar:

1. La Gestalt: Su representante es Max Wertheimer. A través de la afirmación "el todo es más que la simple suma de sus partes", esta teoría expresa que la conciencia es una totalidad organizada, de modo que los pensamientos constituyen percepciones significativas totales y no un conjunto de imágenes agrupadas o expresiones asociadas de estímulo y respuesta.

2. Para la Gestalt, el aprendizaje está basado en la experiencia y la percepción que son el antecedente del comportamiento. El aprendizaje involucra habilidades, actitudes y expectativas que cada sujeto posee y que convierte la misma situación de aprendizaje en un momento único para cada individuo; por esta razón, son de suma importancia las experiencias previas, ya que estas permiten al sujeto organizar los estímulos que recibe de acuerdo a su propia percepción, lo que a su vez caracteriza su comportamiento.

3. Epistemología genética y el Constructivismo: Está representada por el psicólogo francés Jean Piaget, quien expresa que la epistemología es el estudio del paso de un estado de menor conocimiento a estados de conocimiento más avanzado (Facundo, 2013), de modo que lo que interesa es comprender cómo conocemos y no qué conocemos.

Su teoría está basada en el modelo biológico de adaptación aplicado a la inteligencia o conocimiento, de forma que la inteligencia

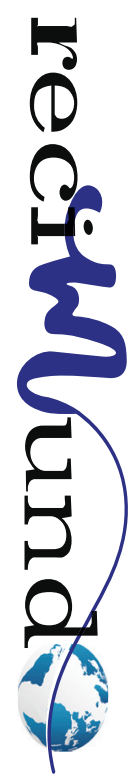


es un tipo de adaptación de un organismo complejo a un medio complejo. El conocimiento es concebido como una adaptación en la que entran en juego estructuras que permiten la interacción entre el sujeto y el medio; esta adaptación implica los mecanismos de asimilación y acomodación (Cuevas, Feliciano, Miranda, \& Catalán, 2015).

Entendemos la asimilación cognitiva como la incorporación del objeto por parte del sujeto mediante esquemas cognitivos; por su parte, la acomodación actúa paralela y complementariamente a la asimilación, permitiendo un ajuste de la estructura cognitiva del organismo (sujeto) a las características del medio. Es necesario tomar en cuenta que lo que el sujeto conoce (objeto) forma parte de su estructura cognitiva a través de la asimilación y acomodación, el objeto es conocido por la interpretación a partir de los esquemas cognitivos; sin embargo, estos esquemas, con la incorporación del nuevo conocimiento, vuelven a modificarse y reorganizarse.

1) Constructivismo: Esta teoría entiende el conocimiento como una construcción, dado que el sujeto no copia la realidad que percibe, sino que selecciona la información que recibe, la interpreta y organiza según sus propios esquemas cognitivos.

Se entiende entonces por Constructivismo a la construcción del conocimiento por parte del sujeto en base a los conocimientos que ya posee, en una continua interacción entre el ambiente y sus propias características cognitivas.

El psicólogo cuyas teorías se incluyen en el Constructivismo es L. Vygotsky; este psicólogo ruso expresa que el aprendizaje en el sujeto es un proceso social, este no es un proceso individual, sino que inicia desde la relación con lo social y pasa luego a ser un proceso interior que permite el desarrollo del sujeto. Uno de los conceptos que explica este proceso es la Zona de Desarrollo
Próximo, este concepto explica la distancia que existe entre el nivel actual de desarrollo real y el desarrollo potencial que el sujeto puede alcanzar ayudado por otra persona; es importante resaltar que Vygotsky realza dos características que aquí se presentan, por un lado el hecho de que el conocimiento inicia en un nivel interpsicológico (entre personas) y concluye en lo intrapsicológico (plano individual), y por otro lado el aprendizaje es mucho más fructífero si se da con ayuda de otro sujeto, es decir, cuando se da en compañía de alguien más: compañeros, hermanos, padres, maestros, etc. (Dueñas, y otros, s.f).

Finalmente, otro aporte al constructivismo lo tenemos en Ausubel quien propuso la teoría del Aprendizaje Significativo según la cual el sujeto que aprende relaciona los conceptos a aprender y les da sentido a partir de sus conocimientos previos.

A partir de las teorías constructivistas, en el ambiente educativo se plantean aproximaciones a situaciones como:

- El desarrollo psicológico del estudiante, particularmente en el plano intelectual y su relación con los ambientes de aprendizaje.

- Identificación y atención a la diversidad de intereses, necesidades y motivaciones de los estudiantes en relación con el proceso enseñanza-aprendizaje.

- Replanteamiento de los contenidos curriculares orientados a conseguir aprendizajes significativos.

- Reconocimiento de la existencia de diferentes estilos de aprendizaje, atendiendo a aspectos intelectuales, afectivos y sociales.

- Creatividad en la selección, organización y de estrategias de aprendizaje.

- Importancia de promover la interacción entre el docente y estudiantes, así como entre los alumnos mismos, mediante el uso de estrategias de aprendizaje cooperativo. 
- Revalorización del papel del docente, como transmisor, guía o facilitador del aprendizaje y como mediador del mismo.

En conclusión, cada sujeto, y en sentido estricto cada estudiante, es el responsable de construir su conocimiento, es una tarea insustituible; en ese sentido, el estudiante no es considerado un simple receptor de un conocimiento dado desde un docente, sino que el docente cumple un papel de mediador del conocimiento y es el estudiante quien selecciona y organiza la información que recibe dándole un significado y sentido propios (Dueñas, y otros, s.f)

\section{Metodologías de enseñanza - aprendiza- je}

La práctica educativa está integrada por tres elementos que son el estudiante (aprendizaje), el profesor (enseñanza) y el contenido (metodología); estos elementos a su vez se encuentran contenidos en sistemas más amplios como son la sociedad, la familia, la cultura, etc.

La relación de estos tres elementos debiera darse de forma igual para que el proceso de enseñanza-aprendizaje avance; sin embargo, en esta relación siempre tendrá más énfasis la participación de dos de ellos, y el tercero tendrá un papel secundario; pues bien, en pedagogía el éxito está en que se logre una relación privilegiada entre dos sin excluir al tercero. De aquí surgen tres modelos de pedagógicos en base a la relación que se privilegie (Latorre \& Seco, 2013):

- $\quad$ Modelo de enseñanza = privilegia el eje Profesor-Contenidos

- Modelo de aprendizaje = privilegia el eje Estudiante-Contenidos

- Modelo de formación = privilegia el eje Profesor-Estudiante

- En la intervención educativa el estudiante es considerado el sujeto principal de la educación que es el proceso de ac- ción-comunicación entre sujetos. Por su parte el docente es el agente de la educación, es el mediador entre el estudiante y los contenidos a aprender, de forma que su tarea es provocar el aprendizaje manteniendo el interés de sus discentes y proponiendo actividades que desarrollen en sus estudiantes habilidades y actitudes en pro del aprendizaje; su relación con el estudiantes es la de guía, orientador, tutor, modelo; de aquí que las exigencias actuales al ro del docente requieren de él características que le permitan favorecer el aprendizaje en sus discentes, tal como lo ilustran las tablas.

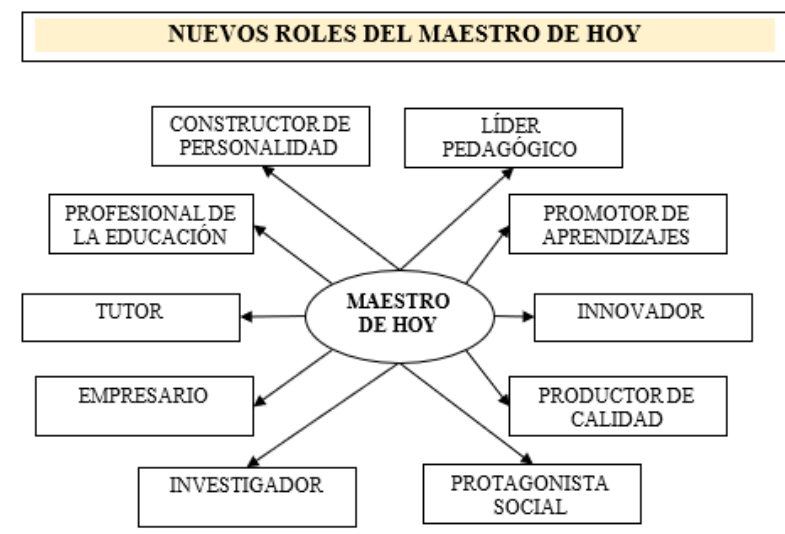

Gráfico 1. Rol del maestro de hoy

Fuente: (Latorre \& Seco, 2013, pág. 11)

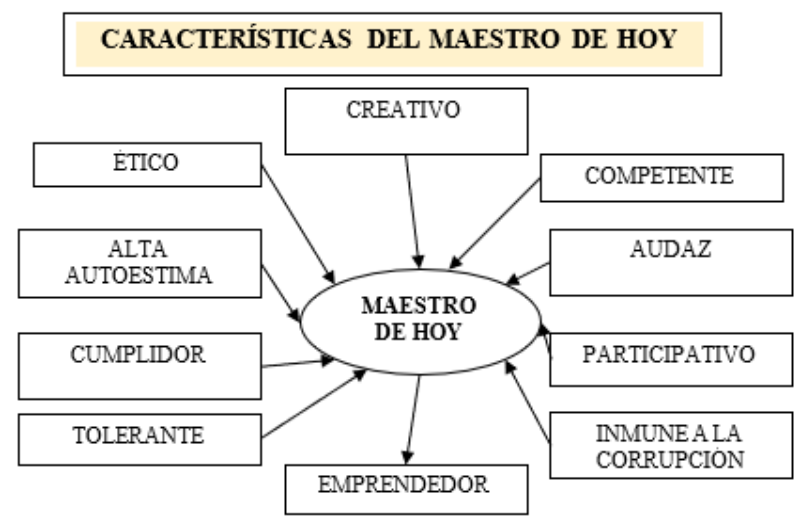

Gráfico 2. Características del maestro de hoy

Fuente: (Latorre \& Seco, 2013, pág. 11) 
Finalmente, los contenidos hacen referencia a los conocimientos, habilidades y procesos mentales, métodos y actitudes del estudiante en relación al aprendizaje, y son el nexo por excelencia entre el estudiante y el conocimiento; es el contenido lo que el docente debe saber proponer y estimular para que el discente logre un aprendizaje significativo; es la metodología de aprendizaje. El conocimiento no es el fin del aprendizaje, sino el medio para que el aprendiz logre habilidades que le permitan aprender a aprender. Una metodología de la enseñanza involucra formas específicas de enseñar, por su parte el método implica una vía y una herramienta específica para transmitir un conocimiento y promover procedimientos para lograrlo entre los estudiantes, alcanzando las metas propuestas por el docente (Albes, y otros, 2013) (Hernández C. , s.f.).

La elección de una metodología específica por parte del docente se ve influenciada por diversos factores como:

- La experiencia previa (como docente y como estudiante)

- El propio concepto de lo que significa enseñar y aprender

- La relación de la metodología escogida con los objetivos que desea alcanzar

- Las características de sus estudiantes (edad, cultura, intereses, nivel de conocimiento, etc.)

- El contenido a enseñar: teórico o práctico
Tabla 5. Relación entre los propios conceptos y la metodología.

\begin{tabular}{|c|c|}
\hline Si entendemos que & $\begin{array}{lll}\text { Entonces nuestra } & \text { metodología } \\
\text { consistirá } & & \\
\end{array}$ \\
\hline $\begin{array}{l}\text { El aprendizaje consiste en } \\
\text { que el alumnado adquiera } \\
\text { una serie de repuestas o } \\
\text { habilidades... }\end{array}$ & $\begin{array}{l}\ldots \text { en centrarse en los ejercicios y la } \\
\text { práctica }\end{array}$ \\
\hline $\begin{array}{l}\text { E1 aprendizaje consiste en } \\
\text { adquirir conocimientos, ... }\end{array}$ & $\begin{array}{l}\text {... en la exposición de contenidos para } \\
\text { que el alumnado los adquiera. }\end{array}$ \\
\hline $\begin{array}{l}\text { E1 aprendizaje consiste en } \\
\text { la construcción del } \\
\text { conocimiento por parte del } \\
\text { alumnado,... }\end{array}$ & $\begin{array}{l}\text {... en la interacción y fomentará la } \\
\text { participación del alumnado, generando } \\
\text { reflexiones sobre la realidad y dándoles } \\
\text { posibilidades de experiencia y contraste } \\
\text { con lo que enseñamos. }\end{array}$ \\
\hline
\end{tabular}

Fuente: (Latorre \& Seco, 2013, pág. 11)

Tabla 6. Modelo de asignación de métodos instruccionales MAM

\begin{tabular}{|c|c|c|c|}
\hline $\begin{array}{c}\text { OBJETIVOS } \\
\text { Conocimiento académico } \\
\text { Científico, formalizados, } \\
\text { acumulados } \mathbf{y} \\
\text { transmitidos } \\
\text { culturalmente }\end{array}$ & $\begin{array}{l}\text { ACTIVO } \\
\text { Profesor }\end{array}$ & $\begin{array}{c}\text { INTERACTIVO } \\
\text { Profesor }\end{array}$ & $\begin{array}{l}\text { PASIVO } \\
\text { Profesor }\end{array}$ \\
\hline $\begin{array}{l}\text { Perspectiva } \\
\text { Logocéntrica }\end{array}$ & $\begin{array}{l}\text { Método } \\
\text { Expositivo- } \\
\text { Conclusivo }\end{array}$ & $\begin{array}{l}\text { Métodos interactivos } \\
\text { Reproductivos: } \\
\text { - Evaluativo } \\
\text { - Calificador } \\
\text { - Tutorial } \\
\text { (monitorizar) }\end{array}$ & $\begin{array}{l}\text { Métodos activos } \\
\text { Reproductivos: } \\
\text { - Entrenamiento } \\
\text { - Prácticas } \\
\text { cerradas }\end{array}$ \\
\hline $\begin{array}{c}\text { Perspectiva } \\
\text { Psicométricas }\end{array}$ & $\begin{array}{l}\text { Método } \\
\text { Expositivo- } \\
\text { Suscitador }\end{array}$ & $\begin{array}{l}\text { Métodos interactivos } \\
\text { Productivos: } \\
\text { - Deductivo } \\
\text { (germinal, } \\
\text { procedimiento } \\
\text { BIG) } \\
\text { - Inductivo } \\
\text { (mapas } \\
\text { conceptuales) } \\
\text { - Horizontal } \\
\text { (socrático, } \\
\text { coloquio, } \\
\text { debate) }\end{array}$ & $\begin{array}{l}\text { Métodos activos } \\
\text { Productivos: } \\
\text { - Investigación y } \\
\text { descubrimientos } \\
\text { - Trabajos } \\
\text { elaborativos }\end{array}$ \\
\hline SUJETOS & $\begin{array}{l}\text { PASIVO } \\
\text { Alumno }\end{array}$ & $\begin{array}{l}\text { INTERACTIVO } \\
\text { Alumno }\end{array}$ & $\begin{array}{l}\text { ACTIVO } \\
\text { Alumno }\end{array}$ \\
\hline
\end{tabular}

Fuente: (Hernández C., s.f., pág. 5)

Una clasificación de las metodologías de enseñanza la encontramos en Hernández (1997; como se citó en Hernández C. , s.f.), en esta clasificación se hace referencia al grado de objetividad del conocimiento ya sea de tipo académico o de experiencias propias, y por otra parte hace referencia al nivel de protagonismo ya sea del docente como del estudiante y los métodos que se involucran en ello, como se muestra en la tabla. 
Tal como se observa en la figura, cuando prevalece la representación del docente, los métodos aplicados son los expositivos; cuando hay participación equilibrada entre docentes y estudiantes, entonces los métodos son interactivos; y cuando la mayor participación la tienen los estudiantes, entonces el método es por descubrimiento.

A continuación, podremos observar una resumida explicación de las estrategias de enseñanza que se han mencionado.

Tabla 7. Metodologías de enseñanza

\begin{tabular}{|c|c|c|c|c|c|}
\hline Metodología & Consiste & Rol activo & Rol pasivo & Se utiliza en & Ventajas \\
\hline Expositiva & $\begin{array}{lr}\text { Exposición } & \text { de } \\
\text { contenidos } & \text { al } \\
\text { alumnado } & \end{array}$ & $\begin{array}{l}\text { Docente tiene } \\
\text { un papel } \\
\text { directivo }\end{array}$ & $\begin{array}{l}\text { Estudiante recibe } \\
\text { información que } \\
\text { docente transmite }\end{array}$ & $\begin{array}{l}\text { Niveles de } \\
\text { educación } \\
\text { superior }\end{array}$ & $\begin{array}{l}\text { - Focalizar el aprendizaje en } \\
\text { grupos grandes } \\
\text { - Requiere menos tiempo de } \\
\text { aprendizaje por parte de } \\
\text { docente porque } \\
\text { información ya esta } \\
\text { sistematizada. } \\
\text { - Requiere menor uso de } \\
\text { material. }\end{array}$ \\
\hline $\begin{array}{l}\text { Interactiva } \\
\text { (método } \\
\text { socrático } \quad 0 \\
\text { comunicativo) }\end{array}$ & $\begin{array}{lr}\text { Transacción } & \text { entre } \\
\text { cociente } & y \\
\text { alumnado } & \text { por } \\
\text { debate o diálogo } \\
\text { para profundizar un } \\
\text { tema }\end{array}$ & $\begin{array}{l}\text { Docentes y } \\
\text { estudiantes }\end{array}$ & & & $\begin{array}{l}\text { - Permite la interacción. Se } \\
\text { puede aplicar en grupos } \\
\text { pequeños. Permite obtener } \\
\text { gran cantidad de } \\
\text { información. }\end{array}$ \\
\hline $\begin{array}{l}\text { Descubrimient } \\
0\end{array}$ & $\begin{array}{l}\text { Utiliza como fuente } \\
\text { de aprendizaje la } \\
\text { experiencia del } \\
\text { sujeto }\end{array}$ & & & & \\
\hline $\begin{array}{l}\text { Método de } \\
\text { descubrimiento } \\
\text { "activo- } \\
\text { reproductivo" }\end{array}$ & $\begin{array}{l}\text { Reproducción de } \\
\text { contenidos }\end{array}$ & $\begin{array}{l}\text { Alumnado } \\
\text { (reproduce } \\
\text { contenidos) }\end{array}$ & $\begin{array}{l}\text { Docente (presenta } \\
\text { modelos } \\
\text { concretos } \\
\text { criterios para que } \\
\text { el estudiante } \\
\text { aplique } \\
\text { practique) }\end{array}$ & & $\begin{array}{l}\text { - Desarrollo de consolidación } \\
\text { de procedimientos. }\end{array}$ \\
\hline $\begin{array}{l}\text { Método de } \\
\text { descubrimiento } \\
\text { "activo- } \\
\text { productivo" }\end{array}$ & $\begin{array}{l}\text { Potencia } \\
\text { pensamiento } \\
\text { productivo }\end{array}$ & $\begin{array}{l}\text { Alumnado } \\
\text { (posibilidad } \\
\text { elaborativa) }\end{array}$ & $\begin{array}{l}\text { Docente (tutoría, } \\
\text { retroalimentación) }\end{array}$ & & $\begin{array}{l}\text { - Permite al alumnado } \\
\text { conocer y practicar técnicas } \\
\text { de investigación en la } \\
\text { realidad. } \\
\text { - Posibilidad de trasladar lo } \\
\text { aprendido a situaciones } \\
\text { diversas. }\end{array}$ \\
\hline
\end{tabular}

Fuente: (Hernández C., s.f., pág. 5) 


\section{Evaluación de estrategias de aprendizaje en estudiantes universitarios}

La educación superior requiere que sus estudiantes adquieran conocimientos propios de su nivel educativo y con autonomía en el aprendizaje. La meta que se desea alcanzar en los estudiantes que egresan de una universidad, oscila entre dos áreas: 1) lo referente a lo abstracto, genérico y de desarrollo personal y 2) lo propio del dominio del conocimiento disciplinario específico de cada carrera.

Dentro de la primera área tenemos el lograr que los estudiantes sean aprendices autónomos, con capacidad de aprender a aprender y de pensamiento crítico. Características de esta área son:

- Analizar ideas y temas de manera crítica.

- Desarrollar habi-lidades intelectuales y de pensamiento.

- Comprender principios y generalizaciones.

Dentro de la segunda área encontramos el dominio de los conocimientos específicos de una profesión a través del desarrollo de las habilidades anteriores. Aquí tiene especial importancia el conocimiento factual de la disciplina junto con el desarrollo de habilidades, estrategias y técnicas que el profesional debe dominar. Como ya hemos visto, las estrategias y técnicas de aprendizaje son las que permiten que el estudiante no solamente logre el aprendizaje requerido de la materia y de la carrera, sino además, lograr que este aprendizaje se dé con autonomía y sea significativo para él (Guzmán, 2011). Esto a su vez, corresponde a una de las características de una educación de calidad en una institución de educación superior. Para lograr conocer cuáles son las estrategias más utilizadas que permiten a los estudiantes lograr un pensamiento crítico y autónomo en su paso por la universidad, es necesario evaluar dichos procesos.

\section{Actividades de aprendizaje}

Corresponden a todas las que permiten que el estudiante se apropie del nuevo conocimiento, están formadas por cuatro componentes de acuerdo al Reglamento de Régimen Académico (RRA): actividades de docencia, actividades autónomas, prácticas de aplicación y experimentación y actividades colaborativas.

Lockwood señala que las actividades de aprendizaje son ejercicios o supuestos prácticos que pretenden que el alumno no se limite a memorizar, sino que esté constantemente aplicando los conocimientos con la finalidad de que los convierta en algo operativo y dinámico. Mediante las actividades se puede guiar y organizar el aprendizaje, ejercitar, afianzar y consolidar lo aprendido, repasar los aspectos destacados de la unidad, controlando el propio aprender, asimilar nuevas ideas integrándolas a lo ya aprendido, favorecer la síntesis interdisciplinar, aplicar los conocimientos a la realidad, generalizar y transferir lo aprendido a otras situaciones, sintetizar, analizar o comparar los componentes de la unidad, leer la realidad y entenderla en profundidad críticamente, buscar creativamente nuevas respuestas interpretativas y, finalmente, motivar el aprendizaje.

Las actividades de aprendizaje entendidas como experiencias por entrar en juego el conocimiento previo que posea el estudiante, se convierten en una estrategia de enseñanza en la medida en que deberán estar bien planeadas y sujetas a la viabilidad de las herramientas tecnológicas con las que se cuente, así como a la temporalidad para la realización de las mismas. (Lockwood, 1978, como se cita en García, 2001, pp. 237-238)

\section{Actividades de aprendizaje asistido por el profesor}

De acuerdo con el RRA (2016) "Correspon- 
den a aquellas actividades que se realizan con el acompañamiento del docente en los diferentes ambientes de aprendizaje. Pueden ser conferencias, seminarios, orientación para estudio de casos, foros, clases en línea en tiempo sincrónico, docencia en servicio realizada en los escenarios laborales, entre otras." (p.10)

"La personalidad del docente es fundamental (...) hoy no solo es responsable de las tareas pedagógicas sino también de las sociales. Su función es cada vez más amplia, compleja y difícil." (Standaert, 2011, p. 91)

\section{Actividades de aprendizaje autónomo}

Según el RRA (2016), "Comprenden el trabajo realizado por el estudiante, orientado al desarrollo de capacidades para el aprendizaje independiente e individual. Este trabaja será diseñado, planificado y orientado por el profesor, para alcanzar los objetivos y el perfil de egreso de la carrera o programa. Son actividades de aprendizaje autónomo, entre otras: la lectura; el análisis y comprensión de materiales bibliográficos y documentales, tanto analógicos como digitales; la generación de datos y búsqueda de información; la elaboración individual de ensayos, trabajos y exposiciones." (pp.10-11) Igualmente, Ruiz (2003) destaca que el desarrollo del aprendizaje autónomo proporciona valores sociales de rectitud, integridad y honradez intelectual; el espíritu crítico y la firmeza se forjan cuando las circunstancias así lo requieren. Para obtener excelentes resultados con esta metodología, es necesario trabajar en habilidades de observación, análisis de la experiencia, comunicación, de lectura y comprensión, elaboración de preguntas, manejo de información y análisis de datos entre otras. (pp. 52-53).

El trabajo autónomo dentro de un entorno virtual de aprendizaje puede fortalecerse mediante la elaboración de glosarios, lecciones, tareas y ejercicios. El estudiante se desenvuelve dentro de una planificación flexible en cuanto a horarios, distancia y escenarios, siempre que cumpla con los objetivos planteados. Es de vital importancia la participación activa con interrogantes y consultas al profesor, con el fin de reforzar conocimientos y de mantener el clima de confianza con el mismo.

\section{Actividades de aprendizaje práctico}

El RRA (2016) define al componente de prácticas de aplicación y experimentación de los aprendizajes, en función de que:

Está orientado al desarrollo de experiencias de aplicación de los aprendizajes. Estas prácticas pueden ser, entre otras: actividades académicas desarrolladas en escenarios experimentales, clínicas jurídicas o consultorios jurídicos gratuitos de las IES, laboratorios, prácticas de campo, trabajos de observación dirigida, resolución de problemas, talleres, entornos virtuales o de simulación, manejo de base de datos y acervos bibliográficos, entre otros. La planificación de estas actividades deberá garantizar el uso de conocimientos teóricos, metodológicos y técnico-instrumentales y podrá ejecutarse en diversos entornos de aprendizaje.

Las actividades prácticas deben ser planificadas y evaluadas por el profesor. (p.10). El afianzamiento del conocimiento dependerá de la frecuencia con la que se realicen estas actividades y la interacción que se consiga con situaciones prácticas reales relativas a la especialidad, apoyadas por la realización de identificación, estructuras anatómicas, la relación de las mismas con su función integral.

\section{Actividades de aprendizaje colaborativo}

De acuerdo con el Reglamento de Régimen Académico (2016), comprenden actividades grupales en interacción con el profesor, incluyendo las tutorías. Están orientadas a procesos colectivos de organización del

\section{2.}


aprendizaje, que abordan proyectos, con temáticas o problemas específicos de la profesión orientadas al desarrollo de habilidades de investigación para el aprendizaje. Son actividades de aprendizaje colaborativo, entre otras: proyectos de integración de saberes, construcción de modelos y prototipos, proyectos de problematización y resolución de problemas o casos; sistematización de prácticas de investigación e intervención, que incluyan metodologías de aprendizaje que promuevan el uso de diversas tecnologías de la información y la comunicación, así como metodologías en red, tutorías in situ o en entornos virtuales. (p.10). Mediante la elaboración de un trabajo en grupo de estudiantes que se ha realizado de manera física, y posteriormente expuesto a sus compañeros; para esto se establece los objetivos del trabajo y las rúbricas de calificación para las temáticas de la asignatura.

\section{Conclusiones}

En el desarrollo de las actividades de las clases es fundamental que el docente sea una guía para el estudiante, pero sobretodos la motivación a través de diferentes estrategias, el uso de materiales didácticos adecuados a la asignatura y de alta calidad han permitido alcanzar niveles de significancia importantes en el grupo de estudio.

Los docentes deben estar en pro de la utilización de programas de trabajo con uso del material didáctico para integrar al estudiante sus conocimientos de mejor manera.

Los docentes con la capacitación constante en el conocimiento de nuevas estrategias didácticas les permitan fortalecer el proceso de enseñanza aprendizaje de sus estudiantes como un proceso integrado a su labor diaria y no como una actividad extra al final de su vida estudiantil.

Las diferentes estrategias para la mejora del proceso enseñanza aprendizaje serán significativas para los estudiantes en la medida en que los docentes sean los primeros en conocerlas, utilizarlas y motivar su utilización permitiendo a los estudiantes experimentar varios caminos de solución aun cuando no sean los tradicionales.

\section{Bibliografía}

Albes, C., Aretxaga, L., Etxebarria, I., Galende, I., Santamaría, A., Uriarte, B., \& Vigo, P. (2013). Orientaciones educativas. Alumnados con altas capacidades intelectuales. Vitoria-Gasteiz, España: Servicio Central de Publicaciones del Gobierno Vasco.

Brunner, J. (1969). Hacia una teoria de la instrucción. Mexico.

Carreño, I. (s.f). Metodologias del aprendizaje. Perú: quebecor Word Perú.

CES, C. D. (17 de 06 de 2014). CONSEJO DE EDUCACION SUPERIOR. Obtenido de http://www.ces. gob.ec/gaceta-oficial/reglamentos

Cuevas, R., Feliciano, A., Miranda, A., \& Catalán, A. (2015). Corrientes teóricas sobre aprendizaje combinado en la educación. Revista Iberoamericana de Ciencias, 75-84.

Díaz-Barriga, F., \& Hernández, G. (2010). Estrategias docentes para un aprendizaje sigificativo. Una interpretación constructivista. México: McGraw-Hill Interamericana.

Dueñas, F., Espinoza, M., López, B., Portillo, E., Rivas, J., \& Soto, A. (s.f). Epistemología del constructivismo. Teoría del conocimiento científico. Epistemología del constructivismo.

Facundo, L. (22 de Noviembre de 2013). Teorías contemporáneas del aprendizaje. Cuernavaca, Morelos, México.

Fierro, M. (2011). El desarrollo conceptual de la ciencia cognitiva. Parte I. Revista Colombiana de Psiquiatría, 519-533.

Garzuzi, V., \& Mafauad, M. (2014). Estilos y estrategias de aprendizaje en alumnos universitarios. Orientación Vocacional, 71-95.

Gravini, M., Cabrera, E., Ávila, V., \& Vargas, I. (2009). Estrategias de enseñanza en docentes y estilos de estrategias de aprendizaje en estudiantes del programa de Psicología de la universidad Simón Bolívar, Barranquilla. Estilos de aprendizaje, 124-139.

Grupo Océano. (2013). diccionario de medicina océano mosby. barccelona, españa: océano. 
Guzmán, J. (2011). La calidad de la enseñanza en educación superior. Perfiles Educativos, 129-141.

Hernández, C. (s.f.). Metodología de enseñanza y aprendizaje en altas capacidades. Superdotación: realidades y formas de abordarlo. España.

Hernández, V., \& Alonso, L. (17 de Septiembre de 2012). El paradigma cognoscitivo en el proceso de enseñanza-aprendizaje. D.F., México. Obtenido de http://es.slideshare.net

Latorre, M., \& Seco, C. (2013). Metodología. Estrategias y técnicas metodológicas. Lima, Perú.

León, A., Risco, E., \& Alarcón, C. (2014). Estrategias de aprendizaje en educación superior en un modelo curricular por competencias. Recista de la Educación Superior, 123-144.

Neufeld, C., Georgen, P., \& Milnitsky, L. (2011). Bases Epistemológicas da Psicologia Cognitiva Experimental. Psicologia: Teoria e Pesquisa, 103-112.
Romero, C. (2012). Fundamentos epistemológicos del conductismo: de la causalidad moderna hacia el pragmatismo. Revista Iberoamericana de Psiocología: Cienic ay Tecnología, 41-48.

Yancen, L., Consuegra, D., Herrera, K., Pacheco, B., \& Díaz, D. (2013). Estrategias educativas utilizadas por los docentes del Programa de Enfermería de una universidad de la ciudad de Barranquilla (Colombia) frente a los estilos de aprendizaje de los estudiantes de este Programa. Salud Uninorte, 405-416.

CES, C. D. (17 de 06 de 2014). CONSEJO DE EDUCACION SUPERIOR. Obtenido de http://www.ces. gob.ec/gaceta-oficial/reglamentos.

Rodriguez, M. L. (2010). La Teoría del Aprendizaje significativo en la perspectiva de la psicologia cognitiva. Barcelona: Ediciones Octaedro.

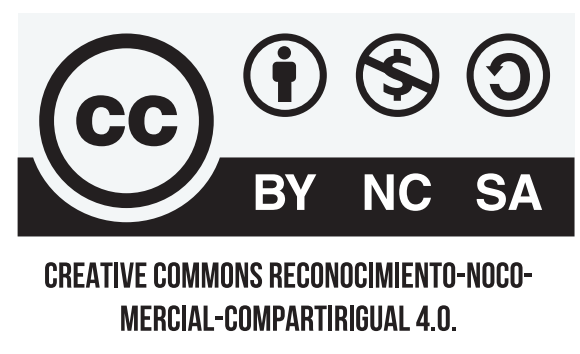

\section{CitAR ESTE ARTICULO:}

Borja Santillán, M. A., Rincón Ríos, T., Santos Jiménez, O. C., \& Gurumendi España, I. E. (2021). Uso del material didáctico para la mejora del proceso de enseñanza aprendizaje en medicina. RECIMUNDO, 5(3), 168-187. https://doi. org/10.26820/recimundo/5.(2).julio.2021.168-187 